(2) Open Access Full Text Article

\title{
Incidence and risk of cardiac toxicities in patients with relapsed and refractory multiple myeloma treated with carfilzomib
}

This article was published in the following Dove Press journal:

Drug Design, Development and Therapy

Fang Zhao'
Bo Yang'
Juan Wang'
Rui Zhang'
Jing Liu'
Fenglei Yin'
Weixing Xu'
Chunyuan He'
'Department of Hematology,
Cangzhou Central Hospital,
Cangzhou, People's Republic of China;
${ }^{2}$ Department of Thoracic Surgery,
Cangzhou Central Hospital, Cangzhou,
People's Republic of China

Correspondence: Fang Zhao Department of Hematology, Cangzhou Central Hospital, No 16, Middle Xinhua Road, Cangzhou 061000, Hebei, People's Republic of China Tel/fax +86 3I7 $20757 / 2$ Email zhaofangczzxyy@aliyun.com
Purpose: Carfilzomib has been approved for use in relapsed and refractory multiple myeloma (RRMM). Cardiac toxicities have been reported with the use of carfilzomib. We aimed to determine the overall incidence and risk of cardiac toxicities in RRMM patients treated with carfilzomib using a meta-analysis.

Methods: We searched several databases for relevant articles. Prospective trials evaluating carfilzomib in RRMM patients with adequate data on cardiac toxicities were included for analysis. Pooled incidence, Peto ORs, and 95\% CIs were calculated according to the heterogeneity of selected studies.

Results: A total of 2,607 RRMM patients from eight prospective trials were included. The pooled incidence of all-grade congestive heart failure (CHF) and ischemic heart disease (IHD) related to carfilzomib in RRMM patients was 5.5\% (95\% CI: 4.3\%-6.9\%) and 2.7\% (95\% CI: $1.1 \%-6.7 \%)$, respectively. In addition, the use of carfilzomib significantly increased all-grade (Peto OR 2.33, 95\% CI: 1.56-3.48, $p<0.001$ ) and high-grade (Peto OR 3.22, 95\% CI: 1.84-5.61, $p<0.001) \mathrm{CHF}$ when compared to controls, whereas there was no significantly increased risk of developing all-grade (Peto OR 1.31, 95\% CI: 0.79-2.18, $p=0.30$ ) and high-grade (Peto OR 1.41, 95\% CI: $0.73-2.72, p=0.31$ ) IHD in RRMM patients receiving carfilzomib.

Conclusion: The use of carfilzomib in RRMM patients significantly increases the risk of developing CHF but not IHD. Clinicians should be cautious about the risk of CHF associated with carfilzomib to maximize the benefits and minimize the toxicities.

Keywords: carfilzomib, cardiac toxicities, clinical trials, meta-analysis, congestive heart failure, ischemic heart disease

\section{Introduction}

The ubiquitin-proteasome system (UPS) plays a vital role in protein degradation involved in many cell events, including cell cycle progression, apoptosis, drug resistance, angiogenesis, and cell differentiation. ${ }^{1-5}$ In addition, many studies have also found that UPS plays a critical role in tumor cell survival; ${ }^{6,7}$ thus, targeting the UPS is a novel treatment strategy for anticancer therapy. ${ }^{5}$ Two major steps are involved in the UPS process: the first step is called the ubiquitination that involves the covalent attachment of ubiquitin(s) to its protein substrate and the second step involves the degradation of the substrate by $20 \mathrm{~S}$ proteasome. ${ }^{8}$ Increased $26 \mathrm{~S}$ proteasome levels and proteasome activity have been detected in several different kinds of malignancies. ${ }^{9-11}$ In addition, high proteasome activity has been found to maintain cancer cell survival because it might aid in protecting against apoptosis pathways and ridding the cell of damaged proteins. ${ }^{9}$ As a result, targeting the proteasome pathway could provide the possibility of developing new opportunities in cancer therapy. 
In the past decades, two proteasome inhibitors (PIs), bortezomib and carfilzomib, have been approved for use in relapsed and refractory multiple myeloma (RRMM) due to their overall survival benefits. ${ }^{12,13}$ However, the UPS has also been involved in maintaining the normal functioning of cardiac myocytes. Thus, inhibition of proteasome function might lead to cardiac insufficiency. Indeed, cardiac toxicities related to PIs have been reported in several published studies. In their meta-analysis, Xiao et al found that the pooled incidence of all-grade and severe cardiotoxicity related to bortezomib in cancer patients was $3.8 \%$ and $2.3 \%$, respectively, although there was no significantly increased risk of cardiotoxicity with bortezomib. ${ }^{14}$ Carfilzomib is a highly selective PI that selectively and irreversibly inhibits the chymotrypsin-like activity of the $20 \mathrm{~S}$ proteasome. In addition, carfilzomib is structurally and mechanistically distinct from bortezomib. Therefore, the cardiac toxicities associated with carfilzomib might be different. However, to our best knowledge, there has been no systematic review to comprehensively assess the cardiotoxicity related to carfilzomib. Therefore, we performed this research to determine the potential cardiotoxicity risk in RRMM patients treated with carfilzomib.

\section{Methods \\ Data source}

Several databases including PubMed, Web of Science, and Cochrane Library were searched for relevant trials. The search key words were carfilzomib, Kyprolis, PR-171, clinical trials, and multiple myeloma (MM). In addition, relevant articles in the reference lists of recent meta-analyses that investigated targeted agents in RRMM patients were also searched. To avoid duplication, only the most complete, recent articles were considered for analysis. All results were input into Endnote X8 reference software (Thomson Reuters, Toronto, ON, Canada) for duplication exclusion and further reference management.

\section{Clinical end point}

Cardiac toxicities were defined by the National Cancer Institute's Common Terminology Criteria for Adverse Events during a clinical trial as a result of exposure to an experimental drug, which had been widely used in cancer clinical trials. ${ }^{15}$ The following adverse outcomes were considered as cardiotoxic events: left ventricular ejection fraction decline or dysfunction, congestive heart failure (CHF) (not specified), and ischemic heart disease (IHD).

\section{Study selection}

The primary objective of the present study was to evaluate the association between carfilzomib treatment and treatment-related cardiac toxicities in RRMM patients; therefore, only prospective Phase II and Phase III trials evaluating carfilzomib in RRMM patients with adequate data on cardiotoxicity were incorporated in the analysis. Phase I trials were omitted due to multiple dose levels and limited sample sizes. Clinical trials that met the following criteria were included: prospective Phase II and Phase III clinical trials in RRMM patients treated with carfilzomib and available data regarding the incidence and causes of cardiac toxicities.

\section{Data extraction}

Two investigators independently conducted data abstraction, and any discrepancy between the reviewers was resolved by consensus. The following information was extracted from each study: first author's name, year of publication, phases of trials, number of enrolled subjects, treatment arms, number of patients in the treatment and control groups, median age, median progression-free survival, adverse outcomes of interest (cardiotoxicity), and dosage of carfilzomib.

\section{Statistical analysis}

The primary summary measures were incidence, Peto ORs, and corresponding 95\% CIs. All statistical analyses were performed using Open MetaAnalyst software version 4.16.12 (Tufts University). For the calculation of incidence, the number of patients with cardiac toxicities in the carfilzomib group and the total number of patients receiving carfilzomib were extracted; the proportion of patients with cardiac toxicities and 95\% CIs were derived for each study. To calculate Peto ORs, patients assigned to carfilzomib were compared only with those assigned to the control treatment in the same trial. We used the Peto method to calculate ORs and 95\% CIs because this method provided the best CI coverage when dealing with low event rates. ${ }^{16}$ Between-study heterogeneity was estimated using the $\chi^{2}$-based $Q$ statistic. ${ }^{17}$ Heterogeneity was considered statistically significant when $P_{\text {heterogeneity }}$ was $<0.1$. The presence of publication bias was evaluated using the Begg's and Egger's tests. ${ }^{18,19}$ A statistical test with a $p$-value $<0.05$ was considered significant.

\section{Results}

\section{Search results}

We performed the systematic review and meta-analysis in accordance with the PRISMA statement. ${ }^{20}$ Our initial search yielded 255 potentially relevant reports. After excluding review articles, Phase I studies, case reports, meta-analyses, and observation studies, a total of eight prospective clinical trials, included three Phase III ${ }^{21-23}$ and five Phase II trials, ${ }^{24-28}$ 
Table I Baseline characteristics of included trials

\begin{tabular}{|c|c|c|c|c|c|c|}
\hline Study & Phase & Total & Treatment regimens & $\begin{array}{l}\text { Median } \\
\text { age (years) }\end{array}$ & $\begin{array}{l}\text { Median PFS } \\
\text { (months) }\end{array}$ & $\begin{array}{l}\text { Median duration } \\
\text { of carfilzomib }\end{array}$ \\
\hline Siegel et al ${ }^{27}$ & II & 266 & Carfilzomib $20 / 27$ mg/m² & 63 & 15.6 & 3.0 \\
\hline Vij et $\mathrm{al}^{26}$ & II & 164 & Carfilzomib $20 \mathrm{mg} / \mathrm{m}^{2}$ & 65 & NR & 7.0 \\
\hline Jagannath et $\mathrm{a}^{28}$ & II & 46 & Carfilzomib $20 \mathrm{mg} / \mathrm{m}^{2}$ & 63.5 & 3.5 & NR \\
\hline Badros et $\mathrm{al}^{25}$ & II & 50 & Carfilzomib $20 \mathrm{mg} / \mathrm{m}^{2}$ followed by $27 \mathrm{mg} / \mathrm{m}^{2}$ & 64 & NR & NR \\
\hline Korde et $\mathrm{a}^{24}$ & II & 45 & Carfilzomib $20 / 36 \mathrm{mg} / \mathrm{m}^{2}+$ lenalidomide+dexamethasone & 60 & NR & 16 \\
\hline \multirow[t]{2}{*}{ Dimopoulos et $\mathrm{al}^{22}$} & III & 929 & Carfilzomib $20 \mathrm{mg} / \mathrm{m}^{2}+$ dexamethasone & 65 & 18.7 & 9.3 \\
\hline & & & Bortezomib+dexamethasone & 65 & 9.4 & 6.3 \\
\hline \multirow[t]{2}{*}{ Hajek et $\mathrm{a}^{21}$} & III & 315 & Carfilzomib 20 mg/m² & 63 & 3.7 & 3.8 \\
\hline & & & Low-dose corticosteroids & 66 & 3.3 & 2.5 \\
\hline \multirow[t]{2}{*}{ Stewart et $\mathrm{a}^{23}$} & III & 792 & Carfilzomib $20 \mathrm{mg} / \mathrm{m}^{2}+$ lenalidomide+dexamethasone & 64 & 26.3 & 20.5 \\
\hline & & & Lenalidomide+dexamethasone & 65 & 17.6 & 13.3 \\
\hline
\end{tabular}

Abbreviations: PFS, progression-free survival; NR, not reported.

were included (Table 1). In total, 2,607 RRMM patients were pooled for analysis. A flowchart showing the study selection process is shown in Figure 1. The baseline characteristics of patients and included studies are shown in Table 1.

\section{Overall incidence of cardiotoxicity}

For the incidence analysis, we considered arms receiving either carfilzomib monotherapy or carfilzomibbased combination. A total of 2,607 MM patients were available for analysis. The reported all-grade $\mathrm{CHF}$ incidence ranged between $3.8 \%$ and $8.2 \%$. The pooled incidence of all-grade CHF in RRMM patients was 5.6\% (95\% CI: 4.3\%-6.9\%; Figure 2A). There was significant heterogeneity among included trials; thus, we performed the meta-analysis using a random effects model. As for all-grade IHD, a total of 1,126 patients from three trials were included. All-grade IHD incidence ranged between $0.8 \%$ and $5.8 \%$. The overall incidence of all-grade IHD in RRMM patients treated with carfilzomib was $2.7 \%(95 \%$ CI: $1.1 \%-6.7 \%$; Figure $2 \mathrm{~B}$ ).

\section{Relative risk of cardiotoxicity}

Three randomized studies were available to calculate the Peto OR of all-grade and high-grade CHF in RRMM patients assigned to carfilzomib versus controls in the same trial. The present study showed that the summary Peto OR of

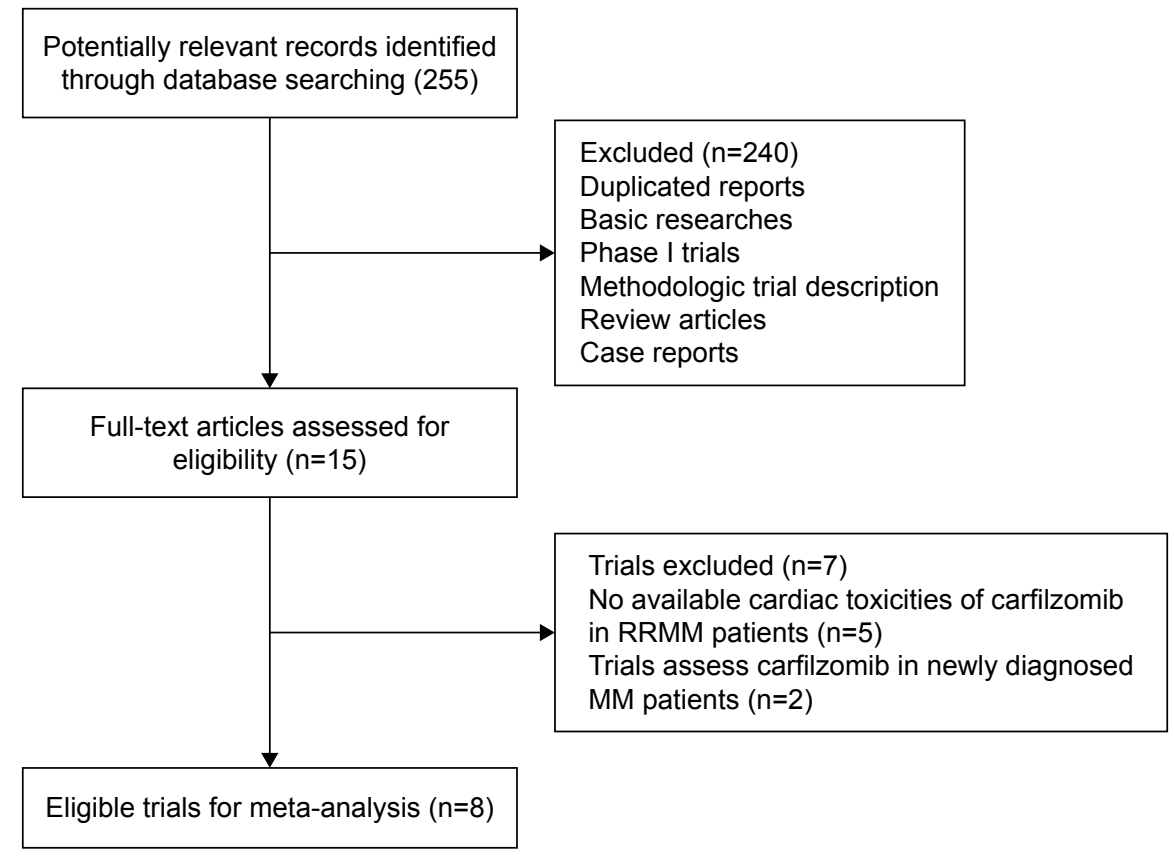

Figure I Flowchart of trial selection process in the meta-analysis.

Abbreviations: RRMM, relapsed and refractory multiple myeloma; MM, multiple myeloma. 
A

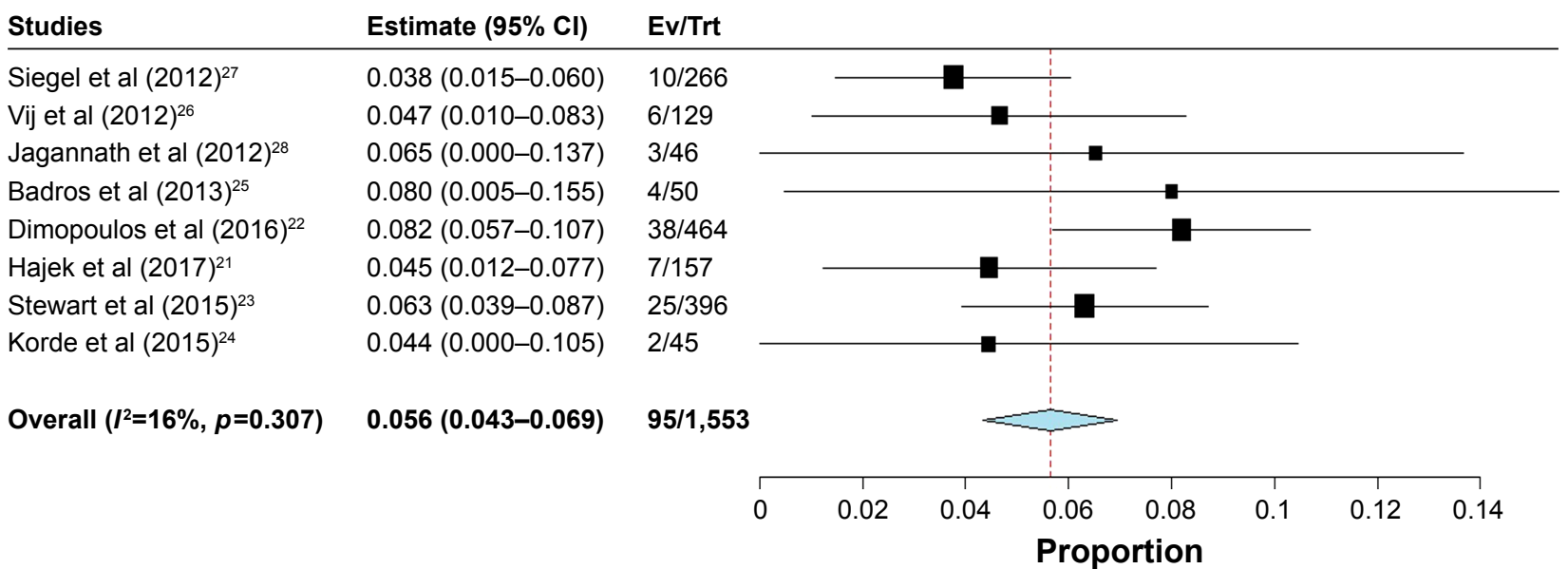

B

\section{Studies}

Siegel et al $(2012)^{27}$

Dimopoulos et al $(2016)^{22}$

Stewart et al $(2015)^{23}$

Overall $\left(I^{2}=83 \%, p=0.003\right)$
Estimate $(95 \% \mathrm{Cl}) \quad$ Ev/Trt

$0.008(0.002-0.030) \quad 2 / 266$

$0.026(0.015-0.045) \quad 12 / 464$

$0.058(0.039-0.086) \quad 23 / 396$

$0.027(0.011-0.067)$
$37 / 1,126$

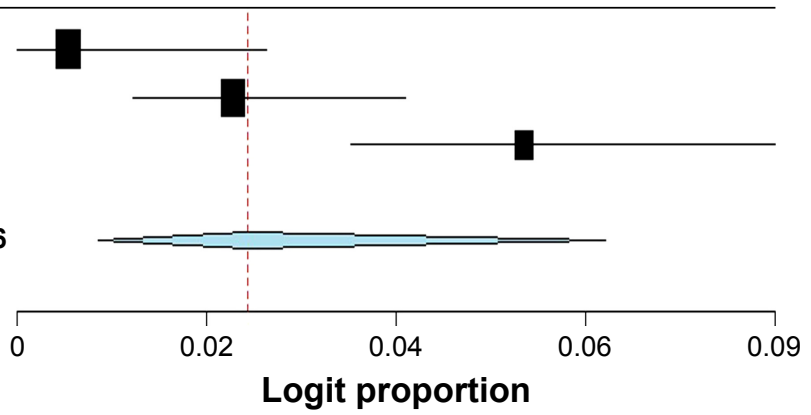

Figure 2 Forest plot for meta-analysis of incidence of $(\mathbf{A})$ all-grade and (B) high-grade CHF in RRMM patients receiving carfilzomib. Abbreviations: $\mathrm{CHF}$, congestive heart failure; Ev, events; RRMM, relapsed and refractory multiple myeloma; Trt, treatment.

all-grade and high-grade $\mathrm{CHF}$ in carfilzomib versus controls arms was 2.33 (95\% CI: 1.56-3.48, $p<0.001$; Figure 3A) and 3.22 (95\% CI: 1.84-5.61, $p<0.001$; Figure 3B), respectively, suggesting a nearly three times higher risk of developing CHF associated with carfilzomib compared with controls. The test for heterogeneity was not significant $\left(I^{2}=29 \%\right.$, $p=0.24$ and $I^{2}=0 \%, p=0.38$, respectively).

Two randomized trials were available to calculate the Peto OR of all-grade and high-grade IHD. The present study showed that the summary Peto OR of all-grade and high-grade IHD in experimental versus control arms was nonsignificant (all-grade: Peto OR 1.31, 95\% CI: 0.79-2.18, $p=0.30$; highgrade: Peto OR 1.41, 95\% CI: $0.73-2.72, p=0.31)$. The test for heterogeneity was not found to be significant $\left(I^{2}=30 \%\right.$, $p=0.25$ and $I^{2}=0 \%, p=0.70$, respectively).

\section{Discussion}

MM is a clonal neoplastic proliferation of plasma cells in the bone marrow associated with the production of monoclonal immunoglobulins in the blood or urine.
Although great advances have been achieved by the introduction of novel drugs, a majority of MM patients eventually relapse, and the prognosis of these patients who are refractory to immunomodulatory drugs and/or bortezomib is very poor, highlighting the need for additional agents. In the past years, carfilzomib has been approved for use in heavily pretreated MM patients due to its survival benefits. However, the risk of cardiac toxicities is a serious concern with the increased application of carfilzomib in the treatment of RRMM patients.

As far as we know, our study is the first meta-analysis to specially assess the risk of cardiac toxicities in RRMM patients treated with carfilzomib. With available evidence from published clinical trials, the present research included 2,607 MM patients from eight prospective clinical trials. Our pooled results suggested that the incidence of all-grade $\mathrm{CHF}$ and IHD related to carfilzomib was 3.8\% (95\% CI: $2.6 \%-5.6 \%$ ) and $2.3 \%$ (95\% CI: $1.6 \%-3.5 \%)$, respectively. In addition, we found that the use of carfilzomib was associated with a significantly increased risk of developing 
A

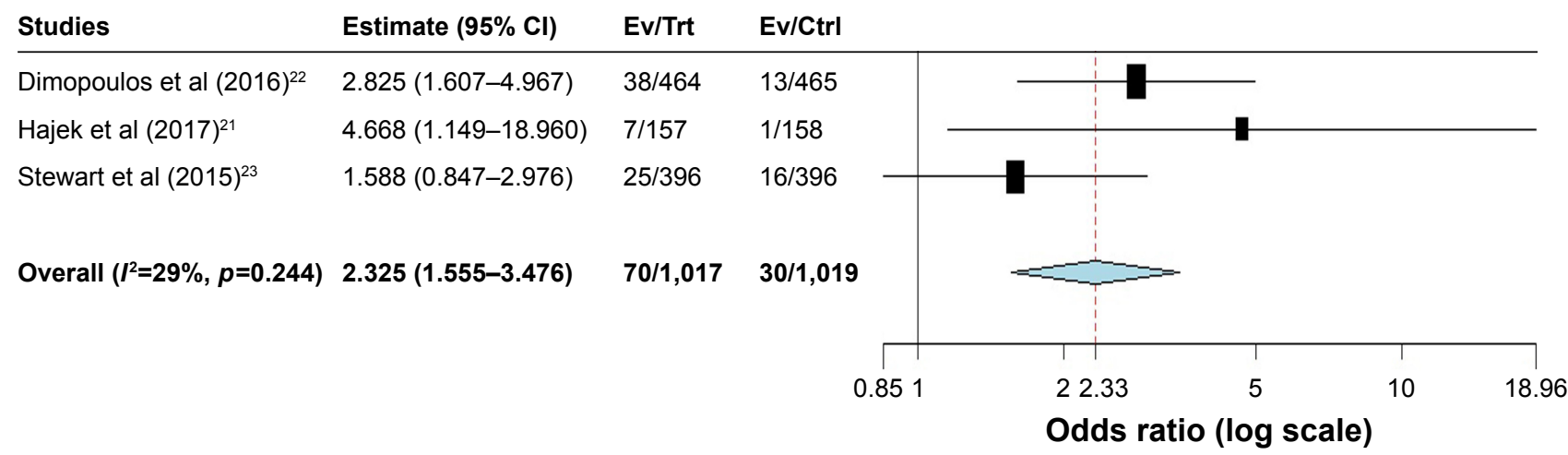

B

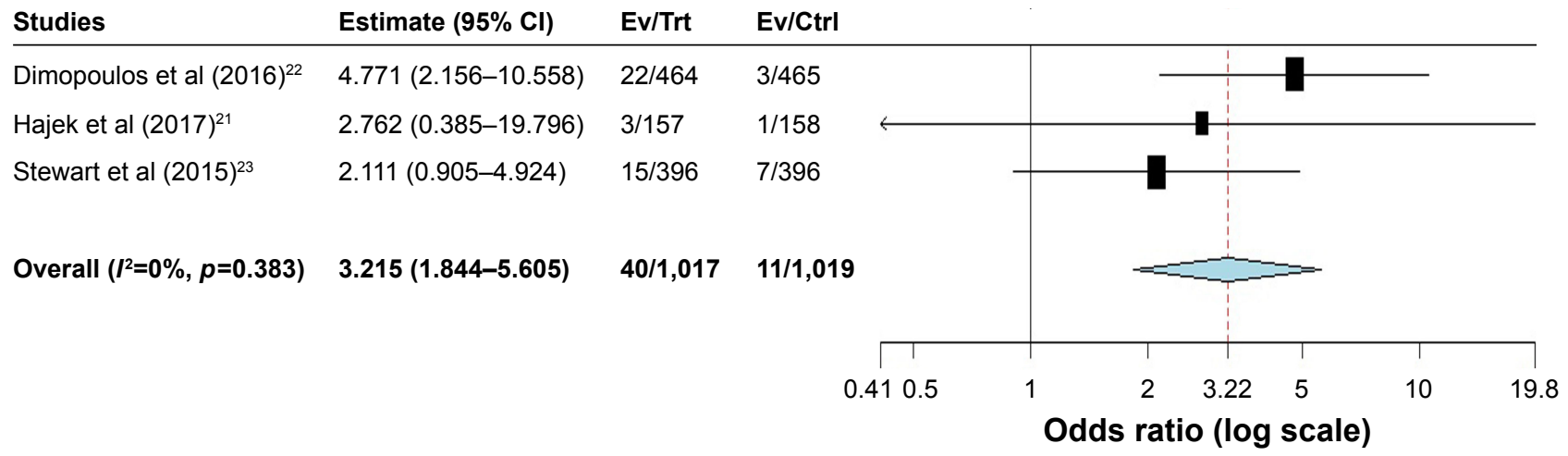

Figure 3 Relative risk of carfilzomib-associated (A) all-grade and (B) high-grade CHF in RRMM patients receiving carfilzomib.

Abbreviations: CHF, congestive heart failure; Ctrl, control; Ev, events; RRMM, relapsed and refractory multiple myeloma; Trt, treatment.

all-grade (Peto OR 2.33, $p<0.001$ ) and high-grade (Peto OR $3.22, p<0.001) \mathrm{CHF}$ when compared with controls, whereas there was no significantly increased risk of developing allgrade (Peto OR 1.31, $p=0.30$ ) and high-grade (Peto OR $1.41, p=0.31$ ) IHD in RRMM patients receiving carfilzomib. However, only two prospective randomized controlled trials were included to investigate the risk of IHD associated with carfilzomib; thus, the power to investigate the risk was small. Nevertheless, as carfilzomib is used extensively in the routine treatment of RRMM patients, it is important for physicians to recognize the risk of $\mathrm{CHF}$ including cardiac ischemia during the administration of carfilzomib therapy, especially for patients with preexisting coronary cardiac disease. Further studies are recommended to determine the risk factors and underlying mechanisms for risk reduction. In addition, the risk of cardiac toxicities might be varied according to the length of carfilzomib treatments. Data on the length of treatment are reported in three randomized controlled trials. The result indicates that the relative risk of cardiac toxicities is not significantly associated with the duration of carfilzomib treatment. The highest risk of cardiac toxicities is observed in the trials conducted by Hajek et al, ${ }^{21}$ but the treatment duration of carfilzomib is shorter than the other trials (Table 1).

As proteasome plays a critical role in the myocardium, particularly in the context of cardiac stress, an elevated risk of cardiovascular toxicity has been observed with other PIs, including bortezomib. Because carfilzomib is a more potent, irreversible PI, its use could pose a greater cardiovascular risk when compared to bortezomib. A direct comparison of cardiovascular toxicities between carfilzomib and bortezomib is available in the ENDEAVOR trial. ${ }^{22}$ In that trial in the setting of relapsed/refractory disease, cardiac event of any grade was reported in $12 \%$ of patients in the carfilzomib arm compared with $4 \%$ in the bortezomib arm.

Currently, the detailed mechanism of cardiotoxicity associated with carfilzomib is still unknown. Several different theories regarding the pathogenesis of cardiotoxicity have been postulated. It has been found that reduced proteasome activity might increase apoptosis in smooth muscle cells, leading to atherosclerotic plaque instability. ${ }^{29,30}$ This could increase the propensity of the atherosclerotic plaque to rupture, which may lead to ischemic complications. In addition, in an in vitro study, bortezomib has been found to cause 
significant structural abnormalities in the mitochondria of the cardiomyocytes, which could result in reduced cardiac contractility. ${ }^{31}$ As a result, PIs treatment might be associated with significant left ventricular contractile dysfunction.

According to carfilzomib prescribing information, close monitoring is recommended for patients with signs or symptoms of cardiac events including heart failure, myocardial infarction, IHD, and hypertension. If patients experience a grade 3 or 4 cardiac event, carfilzomib should be held until resolution to grade 1 , and a dose reduction may be necessary if therapy is continued. Patients with a cardiac history or advanced age ( $\geq 75$ years) should be closely evaluated prior to and while receiving carfilzomib.

Several limitations are needed to be considered in this analysis. First, our study is a meta-analysis of published data but not a meta-analysis of individual patient data. Thus, patient variables could not be incorporated into the analysis. Second, studies included in our analysis are conducted at various institutions by distinct investigators and may have potential bias in reported incidences or specifications of cardiac toxicities. Finally, cardiac toxicities are not the primary end point in all included trials, and the process of attribution of cardiac toxicities' causality might be a potential source of bias.

\section{Conclusion}

The present research shows that the use of carfilzomib in RRMM patients significantly increases the risk of developing CHF but not IHD in comparison with controls. It is important for physicians and patients to appropriately recognize the risks as well as the benefits associated with carfilzomib treatment with regular monitoring of cardiac toxicities during the treatment.

\section{Disclosure}

The authors declare that they have no conflicts of interest in this work.

\section{References}

1. Mitsiades N, Mitsiades CS, Richardson PG, et al. The proteasome inhibitor PS-341 potentiates sensitivity of multiple myeloma cells to conventional chemotherapeutic agents: therapeutic applications. Blood. 2003; 101(6):2377-2380.

2. Lu G, Punj V, Chaudhary PM. Proteasome inhibitor Bortezomib induces cell cycle arrest and apoptosis in cell lines derived from Ewing's sarcoma family of tumors and synergizes with TRAIL. Cancer Biol Ther. 2008; 7(4):603-608.

3. Galimberti S, Canestraro M, Pacini S, et al. PS-341 (Bortezomib) inhibits proliferation and induces apoptosis of megakaryoblastic MO7-e cells. Leuk Res. 2008;32(1):103-112.
4. Baiz D, Pozzato G, Dapas B, et al. Bortezomib arrests the proliferation of hepatocellular carcinoma cells HepG2 and JHH6 by differentially affecting E2F1, p21 and p27 levels. Biochimie. 2009;91(3):373-382.

5. Paramore A, Frantz S. Bortezomib. Nat Rev Drug Discov. 2003;2(8): 611-612.

6. Strous GJ, Govers R. The ubiquitin-proteasome system and endocytosis. J Cell Sci. 1999;112(Pt 10):1417-1423.

7. Ruckrich T, Kraus M, Gogel J, et al. Characterization of the ubiquitinproteasome system in bortezomib-adapted cells. Leukemia. 2009;23(6): 1098-1105.

8. Glickman MH, Ciechanover A. The ubiquitin-proteasome proteolytic pathway: destruction for the sake of construction. Physiol Rev. 2002; 82(2):373-428.

9. Arlt A, Bauer I, Schafmayer C, et al. Increased proteasome subunit protein expression and proteasome activity in colon cancer relate to an enhanced activation of nuclear factor E2-related factor 2 (Nrf2). Oncogene. 2009;28(45):3983-3996.

10. Kumatori A, Tanaka K, Inamura N, et al. Abnormally high expression of proteasomes in human leukemic cells. Proc Natl Acad Sci US A. 1990; 87(18):7071-7075.

11. Chen L, Madura K. Increased proteasome activity, ubiquitin-conjugating enzymes, and eEF1A translation factor detected in breast cancer tissue. Cancer Res. 2005;65(13):5599-5606.

12. VELCADE (bortezomib) for injection [prescribing information]. Cambridge, MA: Millennium Pharmaceutical, Inc; 2003. Available from: http://www.accessdata.fda.gov/drugsatfda_docs/ label/2008/021602s015lbl.pdf. Acceessed August 1, 2013.

13. Herndon TM, Deisseroth A, Kaminskas E, et al. U.S. Food and Drug Administration approval: carfilzomib for the treatment of multiple myeloma. Clin Cancer Res. 2013;19(17):4559-4563.

14. Xiao Y, Yin J, Wei J, Shang Z. Incidence and risk of cardiotoxicity associated with bortezomib in the treatment of cancer: a systematic review and meta-analysis. PLoS One. 2014;9(1):e87671.

15. NCI. Cancer Therapy Evaluation Program. CTC v 2.0 and common terminology criteria for adverse events criteria V3.0 (CTCAE). 2006. Available from: https://ctep.cancer.gov/protocolDevelopment/electronic_applications/ctc.htm\#ctc_40. Accessed April 10, 2018.

16. Kantarjian H, Shah NP, Hochhaus A, et al. Dasatinib versus imatinib in newly diagnosed chronic-phase chronic myeloid leukemia. $N$ Engl J Med. 2010;362(24):2260-2270.

17. Zintzaras E, Ioannidis JP. Heterogeneity testing in meta-analysis of genome searches. Genet Epidemiol. 2005;28(2):123-137.

18. Yusuf S, Peto R, Lewis J, Collins R, Sleight P. Beta blockade during and after myocardial infarction: an overview of the randomized trials. Prog Cardiovasc Dis. 1985;27(5):335-371.

19. Begg CB, Mazumdar M. Operating characteristics of a rank correlation test for publication bias. Biometrics. 1994;50(4):1088-1101.

20. Moher D, Liberati A, Tetzlaff J, Altman DG; PRISMA Group. Preferred reporting items for systematic reviews and meta-analyses: the PRISMA statement. PLoS Med. 2009;6:e1000097.

21. Hajek R, Masszi T, Petrucci MT, et al. A randomized phase III study of carfilzomib vs low-dose corticosteroids with optional cyclophosphamide in relapsed and refractory multiple myeloma (FOCUS). Leukemia. 2017;31(1):107-114.

22. Dimopoulos MA, Moreau P, Palumbo A, et al; ENDEAVOR Investigators. Carfilzomib and dexamethasone versus bortezomib and dexamethasone for patients with relapsed or refractory multiple myeloma (ENDEAVOR): a randomised, phase 3, open-label, multicentre study. Lancet Oncol. 2016;17(1):27-38.

23. Stewart AK, Rajkumar SV, Dimopoulos MA, et al; ASPIRE Investigators. Carfilzomib, lenalidomide, and dexamethasone for relapsed multiple myeloma. $N$ Engl J Med. 2015;372(2):142-152. 
24. Korde N, Roschewski M, Zingone A, et al. Treatment with carfilzomiblenalidomide-dexamethasone with lenalidomide extension in patients with smoldering or newly diagnosed multiple myeloma. JAMA Oncol. 2015;1(6):746-754.

25. Badros AZ, Vij R, Martin T, et al. Carfilzomib in multiple myeloma patients with renal impairment: pharmacokinetics and safety. Leukemia. 2013;27(8):1707-1714.

26. Vij R, Wang M, Kaufman JL, et al. An open-label, single-arm, phase 2 (PX-171-004) study of single-agent carfilzomib in bortezomib-naive patients with relapsed and/or refractory multiple myeloma. Blood. 2012;119(24):5661-5670.

27. Siegel DS, Martin T, Wang M, et al. A phase 2 study of single-agent carfilzomib (PX-171-003-A1) in patients with relapsed and refractory multiple myeloma. Blood. 2012;120(14):2817-2825.
28. Jagannath S, Vij R, Stewart AK, et al. An open-label single-arm pilot phase II study (PX-171-003-A0) of low-dose, single-agent carfilzomib in patients with relapsed and refractory multiple myeloma. Clin Lymphoma Myeloma Leuk. 2012;12(5):310-318.

29. Jancso G, Cserepes B, Gasz B, et al. Effect of acetylsalicylic acid on nuclear factor-kappaB activation and on late preconditioning against infarction in the myocardium. J Cardiovasc Pharmacol. 2005;46(3): 295-301.

30. Versari D, Herrmann J, Gossl M, et al. Dysregulation of the ubiquitinproteasome system in human carotid atherosclerosis. Arterioscler Thromb Vasc Biol. 2006;26(9):2132-2139.

31. Nowis D, Maczewski M, Mackiewicz U, et al. Cardiotoxicity of the anticancer therapeutic agent bortezomib. Am J Pathol. 2010;176(6): 2658-2668.

\section{Publish your work in this journal}

Drug Design, Development and Therapy is an international, peerreviewed open-access journal that spans the spectrum of drug design and development through to clinical applications. Clinical outcomes, patient safety, and programs for the development and effective, safe, and sustained use of medicines are the features of the journal, which has also been accepted for indexing on PubMed Central. The manuscript management system is completely online and includes a very quick and fair peer-review system, which is all easy to use. Visit http://www.dovepress.com/testimonials.php to read real quotes from published authors.

Submit your manuscript here: http://www.dovepress.com/drug-design-development-and-therapy-journal 